Edited by Kiriakos Xenitidis and
Colin Campbell
Contents
- - Lbortion and mental health
- - Neural correlates of formal thought disorder
- Public attitudes towards mental illness
- Is there core diffusion tensor imaging pathology
in schizophrenia?

\section{Abortion and mental health}

The December 2008 issue, with its original papers by Fergusson et $a l,{ }^{1}$ Dingle et $a l^{2}$ and its commentaries, ${ }^{3}$ was of great interest to us. Fergusson et al have overcome some of the methodological problems of previous studies. ${ }^{4}$ Nevertheless, their latest study has weaknesses: the womens' abortion status is not verified objectively, only by self-report. There were 153 abortions in 117 women but insufficient data to distinguish the effects of differing numbers of abortions; it is known that women having more than one abortion may differ in many respects from those having a single abortion. ${ }^{4}$ Also, because of the relatively restrictive law in New Zealand - 'continuance of the pregnancy would result in serious danger ... to the ... mental health of the woman' - some selection bias may have been in operation, allowing only women with more traumatic histories to access abortion. We will not discuss the Dingle et al paper, as its failure to account for pregnancy intention (wantedness and timing) in those giving birth means that the comparator is inappropriate. ${ }^{5}$

The Royal College of Psychiatrists' Position Statement of 14 March 2008 mentions that a full systematic review is needed. This has now been done. ${ }^{5}$ Only four studies fell into the authors' 'good evidence and low risk of bias' category. All four studies showed a neutral effect of abortion on mental health, indicating no significant differences between the study comparison groups. So Fergusson et als study can be regarded as the first good-quality study to show a possible negative effect when attempting to answer the question: what is the relative risk of mental health problems for women who chose abortion compared with those who chose to have a live birth and who reported that the pregnancy was unwanted/initially distressing?

As clinicians working in the field of sexual and reproductive health, we favour the approach of Oates et al. ${ }^{3}$ We are supportive of their idea that abortion is not a psychiatric issue and that the Royal College of Psychiatrists should not develop a guideline on abortion. We would never want to go back to the psychiatric referral hurdle-jumping situation before and immediately after the Abortion Act came into force. ${ }^{6}$ The adverse effects of denied abortion must never be forgotten. ${ }^{7}$ Nevertheless, we do value working in partnership with mental health teams for the benefit of certain women requesting abortion who have a history of mental health problems or persistent ambivalence.

Whether abortion causes harm to women's mental health is a question that is not scientifically testable, as women with unwanted pregnancies cannot be randomly assigned to abortion $v$. abortion denied groups. ${ }^{7}$ It seems inappropriate therefore for Casey to talk of potential litigation against abortion providers for failing to provide information on a possible causal link between abortion and subsequent mental health problems. ${ }^{3}$ All women should have rights to reproductive health and selfdetermination, of which safe and dignified access to abortion services is an important part. $^{8}$

1 Fergusson DM, Horwood LJ, Boden JM. Abortion and mental health disorders: evidence from a 30-year longitudinal study. Br J Psychiatry 2008; 193: 444-51.

2 Dingle K, Alati R, Clavarino A, Najman JM, Williams GM. Pregnancy loss and psychiatric disorders in young women: an Australian birth cohort study. Br J Psychiatry 2008; 193: 455-60.

3 Casey $\mathrm{P}$, Oates $\mathrm{M}$, Jones I, Cantwell R. Invited commentaries on ... Abortion and mental health disorders. Br J Psychiatry 2008; 193: 452-4.

4 American Psychological Association, Task Force on Mental Health and Abortion. Report of the Task Force on Mental Health and Abortion. APA 2008 (http://www.apa.org/pi/wpo/mental-health-abortion-report.pdf).

5 Charles VE, Polis CB, Sridhara SK, Blum RW. Abortion and long-term mental health outcomes: a systematic review of the evidence. Contraception 2008; 78: $436-50$

6 Pare $\mathrm{CMB}$, Raven $\mathrm{H}$. Follow-up of patients referred for termination of pregnancy. Lancet 1970; 1: 635-8.

7 House of Commons Science and Technology Committee. Scientific Developments Relating to the Abortion Act 1967: Volume II (HC 1045-II). TSO (The Stationery Office), 2007 (http://www.publications.parliament.uk/pa/ cm200607/cmselect/cmsctech/1045/1045ii.pdf).

8 Cook RJ, Dickens BM, Fathalla MF. Reproductive Health and Human Rights. Oxford University Press, 2003.

Sam Rowlands, Warwick Medical School, Gibbet Hill Campus, University of Warwick, Coventry CV4 7AL, UK. Email: sam.rowlands@warwick.ac.uk; Kate Guthrie, NHS Hull, UK

doi: 10.1192/bjp.195.1.83

Authors' reply: We would like to thank Rowlands \& Guthrie for their positive comments about our paper. ${ }^{1}$ We do not agree that the Dingle et $a l^{2}$ paper should be dismissed on the grounds that it uses an inappropriate comparison. Although this dismissal is consistent with the opinions stated in the review your correspondents cite, ${ }^{3}$ it reflects a common misunderstanding. There are, in fact, two closely related causal questions that one can ask about abortion and mental health. The first concerns whether or not abortion is an adverse life event that increases risks of mental health problems. Answering this question is important for understanding the extent to which women having abortions are an at-risk population for subsequent mental health problems. The second question concerns whether any mental health risks of abortion are greater or less than the mental health risks of unwanted pregnancies that come to term. Answering this question is important for understanding the extent to which abortion may mitigate or exacerbate any mental health problems associated with unwanted pregnancy. Dingle and colleagues ${ }^{2}$ address the first question by showing that women having abortions are an at-risk population for mental health problems, and that these responses seem similar to those of women who experience pregnancy loss.

Rowlands \& Guthrie suggest that our paper has a number of limitations relating to the assessment of abortion, the number of abortions and the social context of the research. However, these problems have different implications for interpreting our research. Any under-ascertainment of abortion is likely to have the effects of biasing estimates of relative risk downwards (providing that under-ascertainment is statistically independent of mental health outcomes); failure to show the effects of multiple abortions does not threaten the validity of our conclusions, but may call the precision of our conclusions into question; and the sociolegal context within which the research was conducted implies that it 
is important to take pre-abortion mental health into account (which we did).

Rowlands \& Guthrie also suggest that an adequate review of this issue has been conducted by Charles et al. ${ }^{3}$ We do not agree. This review has been criticised on the grounds of investigator bias ${ }^{4}$ and these concerns are supported by the somewhat anomalous conclusions the authors draw. For example, the study by Schmierge \& Russo $^{5}$ using the National Longitudinal Study of Youth (NLSY) data is ranked as one of the four 'good' studies despite the facts that only $40 \%$ of women having an abortion were included, and mental health was measured using a single scale of depression, with this outcome being assessed up to 13 years after the abortion. All of these features will bias results towards the null hypothesis. In addition, Charles et al ${ }^{3}$ failed to distinguish between the different causal questions that may be asked about abortion, and misleadingly dismissed a number of studies showing links between abortion and mental health on the grounds that they did not include an appropriate comparison group.

Finally, Rowlands \& Guthrie claim that conclusions cannot be drawn about the causal effects of abortion on mental health in the absence of randomised controlled trials. We believe that this argument overstates the case and there is now growing evidence for two major conclusions about abortion and mental health. First, exposure to abortion is an adverse life event which is associated with a modest increase in risks of mental health problems. Second, the mental health risks associated with abortion may be larger, and certainly are not smaller, than the mental health risks associated with unwanted pregnancies that come to term.

1 Fergusson DM, Horwood $\mathrm{L}$, Boden JM. Abortion and mental health disorders: Evidence from a 30 year longitudinal study. Br J Psychiatry 2008; 193 : 444-51.

2 Dingle K, Alati R, Clavarino A, Najman JM, Williams GM. Pregnancy loss and psychiatric disorders in young women: an Australian birth cohort study. Br J Psychiatry 2008; 193: 455-60.

3 Charles VE, Polis CB, Sridhara SK, Blum RW. Abortion and long-term mental health outcomes: a systematic review of the evidence. Contraception 2008; 78: $436-50$

4 Ertelt S. Researcher: Johns Hopkins paper biased on abortion-mental health issues. LifeNews.com, 2008; 4 December (http://www.lifenews.com/ nat4629.html).

5 Schmiege S, Russo NF. Depression and unwanted first pregnancy: longitudinal cohort study. BMJ 2005; 331: 1303.

David M. Fergusson, Christchurch Health and Development Study, University of Otago, Christchurch School of Medicine and Health Sciences, PO Box 4345, of Otago, Christchurch School of Medicine and Health Sciences, PO Box 4345,
Christchurch, New Zealand. Email: dm.fergusson@otago.ac.nz; L. John Horwood, Joseph M. Boden, Christchurch Health and Development Study, University of Otago, Christchurch School of Medicine and Health Sciences, Christchurch, New Zealand

doi: 10.1192/bjp.195.1.83a

\section{Longitudinal course of cognition in schizophrenia}

In their meta-analysis, ${ }^{1}$ Szöke et al found significant improvements in most neuropsychological variables, along with well-known potential practice effects, and that semantic verbal fluency holds promise as a suitable cognitive endophenotype in schizophrenia. We acknowledge that this review is a step forward, attempting to merge and quantify the evidence from both naturalistic observational studies ${ }^{2}$ and clinical trials. ${ }^{3}$

We agree with the authors that the current literature is limited by the virtual absence of healthy control groups. Since only 9 out of 53 studies reported longitudinal neurocognitive data for controls, it is difficult to disentangle whether patients' cognitive changes are true improvements or confounded by the non-specific effects of practice-related learning. ${ }^{4}$ The use of healthy individuals from test-retest studies ('external controls') is also problematic and the parallel assessment of controls would rule out the possibility that patients' apparent cognitive stability is not deterioration in disguise, as the authors accurately suggest.

In addition, we would like to highlight other critical issues that may limit the conclusions. First, the authors' choice to lower the minimum study duration to 1 month led to the median test-retest interval being only 4 months, which is shorter than the recommended trial duration to evaluate cognitive changes (e.g. at least 6 months). ${ }^{5}$ This also may have biased the review towards short-term clinical trials intended to improve cognitive deficits, especially with second-generation antipsychotics. Had a more stringent and clinically relevant 1-year follow-up cut-off ${ }^{2}$ been used, only 24 out of 53 studies would have been reviewed. Second, approximately half of the 20 clinical trials previously reviewed ${ }^{3}$ are open, and these are mostly clozapine trials of patients who are treatment-resistant. Significant associations between cognitive change and change in negative symptoms are more likely to occur in these studies than in naturalistic follow-up studies. ${ }^{5}$ Third, we feel the authors missed an opportunity to include the distinction between patients with first-episode or chronic schizophrenia and geriatric patients as a potential moderator variable. This could have contributed to a better understanding of the probably complex cognitive pathways during the lifespan.

Despite the number of longitudinal neurocognitive reports, less is known than was originally supposed about the course of cognition in schizophrenia. Only a small subset $(n=4)$ of longitudinal reports have compared these neurocognitive pathways with those of healthy controls over at least 1 year. When reviews ${ }^{2}$ are not focused on the neurocognitive effects of antipsychotics, stable long-term performances and, in some cases, cognitive gains could be expected, thus offering a rather pessimistic picture for cognitive enhancement. This approach seems more useful for understanding the long-term natural history of cognition in schizophrenia. Conversely, this meta-analysis ${ }^{1}$ relies on short-term data, mostly from clinical trials, ${ }^{3}$ and therefore more likely overestimates the potential for cognitive improvement.

In summary, it would be misleading if the conclusions were regarded as a major leap forward instead of as tentative hypotheses awaiting further investigation. Since the possibility remains that the current findings are more valuable to researchers, a new metaanalysis that takes into consideration these and other limitations might be more helpful for clinicians, patients and caregivers.

1 Szöke A, Trandafir A, Dupont ME, Méary A, Schürhoff F, Leboyer M. Longitudinal studies of cognition in schizophrenia: meta-analysis. Br J Psychiatry 2008; 192: 248-57.

2 Rund BR. A review of longitudinal studies of cognitive functions in schizophrenia patients. Schizophr Bull 1998; 24: 425-35.

3 Woodward ND, Purdon SE, Meltzer HY, Zald DH. A meta-analysis of neuropsychological change to clozapine, olanzapine, quetiapine, and risperidone in schizophrenia. Int J Neuropsychopharmacol 2005; 8: 457-72.

4 Goldberg TE, Goldman RS, Burdick KE, Malhotra AK, Lencz T, Patel RC, et al. Cognitive improvement after treatment with second generation antipsychotic medications in first-episode schizophrenia: is it a practice effect? Arch Gen Psychiatry 2007; 64: 1115-22.

5 Buchanan RW, Davis M, Goff D, Green MF, Keefe RS, Leon AC, et al. A summary of the FDA-NIMH-MATRICS workshop on clinical trial design for neurocognitive drugs for schizophrenia. Schizophr Bull 2005; 31: 5-19.

Vicent Balanzá-Martínez, Department of Medicine, University of Valencia, Blasco Ibáñez, 15, 46010 Valencia, Spain; Email: vicente.balanza@uv.es; Manuel J. Cuesta Psychiatric Unit, Virgen del Camino Hospital, Pamplona; Celso Arango, Psychiatry Department, Adolescent Unit, University Hospital Gregorio Marañón, CIBERSAM, Madrid; Benedicto Crespo-Facorro, University Hospital Marqués de Valdecilla, Department of Psychiatry, School of Medicine, University of Cantabria, CIBERSAM, Santander; Rafael Tabarés-Seisdedos, Section of Psychiatry and Psychological Medicine, Department of Medicine, University of Valencia, CIBERSAM, Valencia, Spain

doi: 10.1192/bjp.195.1.84 